\section{MS44-PI Pb-doped KCl: from adsorption to habit and crystal structure modification Dino Aquilano ${ }^{a}$, Linda Pastero ${ }^{a}{ }^{a}$ Dipartimento di Scienze della Terra, University of Turin, Italy \\ E-mail: dino.aquilano@unito.it}

Many papers dealing with alkali-halides crystal morphology have been published since the Sixties. The relationship between crystal growth or dissolution morphology and the presence of surface specific impurities is commonly recognized. Authors usually attributed morphological changes to the random adsorption of the impurity on specific crystal surfaces. The results of our research clearly show that several morphological changes can be correctly interpreted in terms of 2D-epitaxially absorbed phases [1], [2] leading both to morphological and structural changes. In the case of potassium chloride crystals, both in-situ and ex-situ observations on growing and etched crystals were performed [3] in order to demonstrate that the appearance of the octahedron in the crystal morphology is due to the step bunching effect ascribable to the precipitation of $\mathrm{PbCl}_{2}$ crystallites along the $\mathrm{n}$ steps on the $\{100\}$ forms. Inspired by the Lian et al. [3] detailed "morphodrome" showing the crystal habit and surface modifications of $\mathrm{KCl}$ crystals grown in the presence of $\mathrm{Pb}^{++}$as specific impurity, we performed a new series of growth and dissolution experiments in order to improve the old data. The impurity concentration ranges from 5 to $10000 \mathrm{ppm}$ at constant crystallization temperature. Moreover we tried to enlarge the impurity concentration range, joining the molar ratio of the two known perovskite structures $\left(\mathrm{KCl}: \mathrm{PbCl}_{2}\right.$ and $\left.\mathrm{KCl}: 2 \mathrm{PbCl}_{2}\right)$ in order to evaluate the continuity between morphological and structural changes from the cell distortion (anomalous mixed crystal formation due to impurity adsorption and 2D epitaxy between $\mathrm{KCl}$ and $\mathrm{PbCl}_{2}$ ) to the symmetry breakdown (from the anomalous mixed crystal at higher symmetry to the perovskite structure at lower symmetry).

[1] Pastero, L., Aquilano, D., Costa, E., Rubbo, M. (2005). Journal of Crystal Growth 275, e1625-e1630.

[2] Pastero, L., Aquilano D., Moret, M. (2012) Cryst. Growth Des., 12, 2306-2314.

[3] Lian, L., Tsukamoto, K., Sunagawa, I. (1990). Journal of Crystal Growth 99, 150-155.

Keywords: epitaxy, adsorption/absorption, crystal habit.
MS44-P2 Tuning of micro $\alpha$-quartz crystals morphology by low hydrothermal synthesis methods Dino Aquilano ${ }^{a}$, Linda Pastero $^{a}$, Riccardo Leinardi ${ }^{b}$, Francesco Turci ${ }^{b}$, Mara Ghiazza ${ }^{b}$, Bice Fubini ${ }^{b}{ }^{a}$ Dipartimento di Scienze della Terra, University of Turin, Italy, ${ }^{b}$ Dipartimento di Chimica, University of Turin, Italy

E-mail: dino.aquilano@unito.it

Traditional hydrothermal methods are widely diffused for the synthesis of $\alpha$-quartz [1], [2], [3]. As applied to quartz synthesis, the hydrothermal method requires high $\mathrm{P}, \mathrm{T}$ conditions in order to dissolve the supply material. Quenching the solution leads to the rapid nucleation of quartz, but some amorphous unreacted material usually remains at the end of the growth run [4]. In our experiments we started from a Nametasilicate solution. This method allows to reach higher supersaturation values with respect to the solubility of silica in water or in alkaline solution. The Na-metasilicate solution was polymerized adding different polymerizing agents $(\mathrm{HCl}$, $\mathrm{HNO}_{3}, \mathrm{CO}_{2}$ ). The $\mathrm{pH}$ of the starting silica gel ranges between 10.5 and 7. The experiments were performed in PTFE autoclaves at $210^{\circ} \mathrm{C}$ for variable times $(24,48,72,96$ and 168 hours). Moreover, in order to evaluate the effect of the gel strenght on the quartz crystallization, we performed a series of synthesis at different metasilicate concentrations. In all our experiments we obtained $\alpha$-quartz. Only from experiments carried out at very low metasilicate concentrations, we obtained amorphous silica. Furthermore, starting from different polymerizing agents, we can change the crystal quality and morphology by taking advantage from the good (or bad) epitaxy conditions between quartz and the guest phases in the growing medium. Theoretical epitaxies were calculated to confirm this effect and very good epitaxy conditions between quartz and some Na-carbonate phases were found. No epitaxy was found between nitratine and quartz. These results validate a model of cooperation between phases during the quartz growth that become evident comparing both morphology and surface quality of crystals. Additionally, crystal structure deformations were found in the cases of growth cooperation, whereas, when epitaxy is not allowed, very good crystals from the point of view of structural perfection were obtained (with $\mathrm{HNO}_{3}$ as polymerizing agent).

11] Hosaka, M., Taki, S. (1981).J. Crys. Growth, 52/2, 837-842.

[2] Hosaka, M., Taki, S. (1981). J. Crys. Growth, 51, 640-642.

[3] Hosaka, M., Miyata T. (1993). Mat. Res. Bull., 28, 1201-1208.

[4] Jiang, X., Jiang, Y.B., Brinker C. J. (2011) Chem. Commun., 47, 7524-7526

Keywords: -quartz; growth morphology; hydrothermal synthesis. 